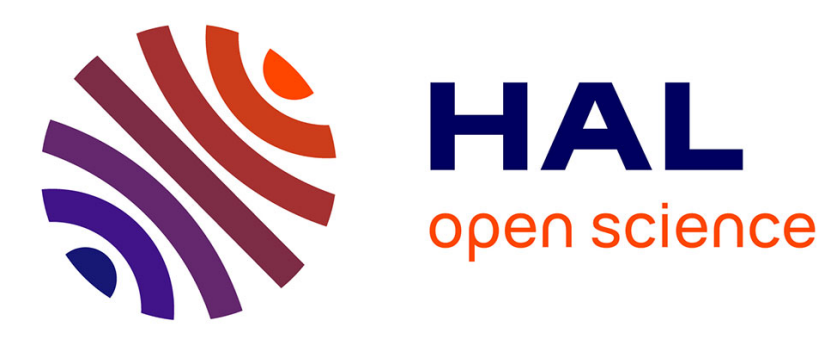

\title{
Analytical model of double-lap bonded joints subjected to impact loads
}

Rachad Hazimeh, Khaled Khalil, Georges Challita, Ramzi Othman

\section{To cite this version:}

Rachad Hazimeh, Khaled Khalil, Georges Challita, Ramzi Othman. Analytical model of double-lap bonded joints subjected to impact loads. International Journal of Adhesion and Adhesives, 2015, 57, pp.1-8. 10.1016/j.ijadhadh.2014.09.004 . hal-03286599

\section{HAL Id: hal-03286599 \\ https://hal.science/hal-03286599}

Submitted on 28 Jul 2021

HAL is a multi-disciplinary open access archive for the deposit and dissemination of scientific research documents, whether they are published or not. The documents may come from teaching and research institutions in France or abroad, or from public or private research centers.
L'archive ouverte pluridisciplinaire HAL, est destinée au dépôt et à la diffusion de documents scientifiques de niveau recherche, publiés ou non, émanant des établissements d'enseignement et de recherche français ou étrangers, des laboratoires publics ou privés.

\section{(c)(1)}

Distributed under a Creative Commons Attribution| 4.0 International License 


\title{
Analytical model of double-lap bonded joints subjected to impact loads
}

\author{
Rachad Hazimeh $^{\mathrm{a}}$, Khaled Khalil ${ }^{\mathrm{b}}$, Georges Challita ${ }^{\mathrm{b}}$, Ramzi Othman ${ }^{\mathrm{c}, *}$ \\ a LUNAM Université, Ecole Centrale de Nantes, Institut de Recherche en Génie Civil et Mécanique, UMR CNRS 6183, BP 92101, 44321 Nantes cedex 3, \\ France ${ }^{\mathrm{b}}$ Equipe MMC, Université Libanaise, Faculté de génie, Campus Hadath, Beyrouth, Lebanon \\ ${ }^{\mathrm{c}}$ Mechanical Engineering Department, Faculty of Engineering, King Abdulaziz University, P.O. Box 80248, Jeddah 21589, Saudi Arabia
}

\begin{abstract}
A stress analysis of adhesively bonded double lap joints having half-infinite lengths was performed using a half-closed-form approach. The approach is based on an improved shear-lag model. Thus normal deformations and shear deformations were considered inside the adherends. Differential equations governing adherends-interfaces displacements were extracted from the dynamic equilibrium equations. Laplace transform was used to solve the differential equations. The stress variation with respect to time at the edge of the adhesive layer was investigated. Transfer function between applied load and adhesive edge shear stress was extracted. Impulse response was deduced using the inverse Laplace's transform of the transfer function. Impulse response appeared to be a zero-order Bessel function. The indicial response of the joint can be calculated by the integration of the impulse response over time. The model was validated for different substrates' materials.
\end{abstract}

\section{Introduction}

Adhesively bonded joints are increasingly used in various industrial sectors. They are mainly used in transport vehicles and electronic devices. They are used to assemble substrates of similar and dissimilar materials. The adhesive joints may undergo various loads: in-plane or transverse; static, harmonic or impact. The impact behaviour of adhesively-bonded joints can be approached by either experimental [1-4], numerical [5-7] or analytical $[8,9]$ techniques.

Over the decades, several theoretical approaches have been performed to analyse the response of adhesively bonded joints [10]. The pioneer was Volkersen [11] who proposed the shear-lag model for single-lap joints. The shear-lag assumes that the substrates undergo only normal stresses and the adhesive is subjected to pure shear stresses. This shear stress is proportional to the relative displacement of substrates. The model was expanded to double lap joints by De Bruyne [12]. Goland and Reissner [13] considered the substrates as beams bonded together and subjected to both normal and shear stresses. Oplinger [14] extended this approach to allow for adhesive deflection. Hart Smith [15] worked with the original shearlag model taking into consideration the elasto-plastic behaviour of the adhesive. Tsai et al. [16] improved the shear-lag model to include

\footnotetext{
* Corresponding author.

E-mail addresses: Rachad.Hazimeh@ec-nantes.fr (R. Hazimeh) Khkhalil@ul.edu.lb (K. Khalil), George_Challita@yahoo.com (G. Challita), rothman1@kau.edu.sa (R. Othman).
}

shear deformation in substrates, which allows a better description of joints made with adherends having low shear stiffness.

Since the pioneer work of Volkersen [11], significant interests have been expressed in the analytical solution approximating the quasi-static response of adhesively bonded joints [10]. Very few works dealt with the dynamic response. In terms of the harmonic response, Vaziri et al. $[17,18]$ studied the response to peeling loads. Vaziri and Nayeb-Hashemi studied the response due to harmonic torsional [19] and axial [20] loads. In Ref. [20], the original shearlag model [11] was applied to predict harmonic stresses in tubular joints. Challita and Othman [21] extended the improved shear-lag model developed in Ref. [16] to approximate the harmonic response of double-lap bonded joints. To the best of the author's knowledge, only two works have dealt with the analytical modelling of the impact response of bonded joints. Namely, Pang et al. [8] were interested in single lap composite joint. In order to catch the impact response they considered the adhesive as a spring-mass model. Recently, Sato [9] investigated the shear-lag model subjected to impact loadings for half-strap infinite length joint, and found that the system impulse response can be described by a zero-order Bessel function.

This paper aims at developing an analytical solution of the impact response of double-lap bonded. For this purpose, the harmonic model developed by Challita and Othman [21], which takes into consideration the substrates' shear stiffness, will be extended to consider impact loadings. Laplace transforms as suggested by Sato [10] will be used to move from the harmonic to the impact response. 


\section{Theoretical approach}

\subsection{Problem definition and assumptions}

In this work, we aimed at predicting the stress response in an infinite double-lap joint due to an impulsive and indicial stress wave transmitted to the joint from an infinite middle substrate (Fig. 1). The thickness for the upper and lower substrates, for the middle substrate and adhesive layer are " $e$ ", " $2 e$ " and " $e_{0}$ ", respectively. The three substrates were considered from the same material. Both, the adhesive and substrates were assumed elastic wherein $G, E$ and $\rho$ are the substrates' shear modulus, Young's modulus and density, respectively, and $G_{0}$ is the adhesive shear modulus.

The improved shear-lag model [16,21] was applied here to represent the stress state in the double-lap joint (Fig. 2). Namely, the substrates were subjected to axial and shear stresses whereas only shear stress in the adhesive layer was allowed. The shear stress in the substrates was assumed linear in terms of the transverse (through-thickness or $y$-direction) coordinate and proportional to the shear deformation. The improved shear-lag model takes into account the inertia of the substrates. On the opposite, the adhesive layer inertia was neglected.

The model is semi-infinite in the axial direction or $x$-direction. As a boundary condition, a stress wave was assumed at the virtual interface of the middle substrate at $x=0$ as it will be shown in Section 2.4. No wave reflections were assumed at the infinity $(x=\infty)$. Due to symmetry, only the upper adherend and half of middle substrate were considered. The shear stress at the longitudinal axis of symmetry $(y=0)$ vanishes. This is also the case for the upper surface of the upper adherend $\left(y=2 e+e_{0}\right)$.

Henceforth, the subscripts "c", "a" and " 0 " were used for the middle substrate, upper substrate and adhesive layer, respectively. Hence, $u_{c}$ and $u_{a}$ denote middle and upper adherends displacements. Moreover, $\tau_{0}$ holds for the adhesive shear stress, which is assumed constant through the thickness of the adhesive. $\tau_{a}$ and $\tau_{c}$ hold for the shear stresses inside the upper and middle substrates, respectively. The axial or normal forces applied on upper and middle substrates' cross-section are denoted $T_{a}$ and $T_{c}$.

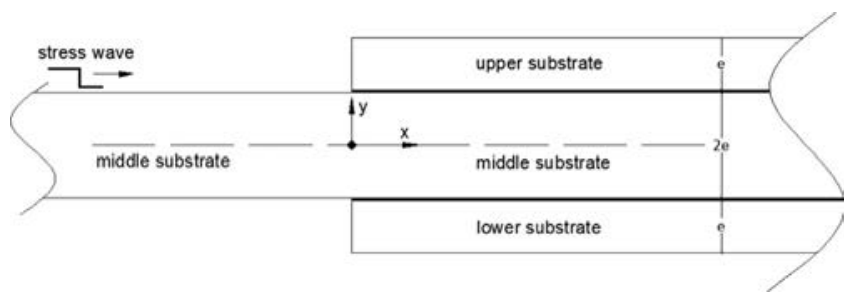

Fig. 1. Model geometry with loading condition.
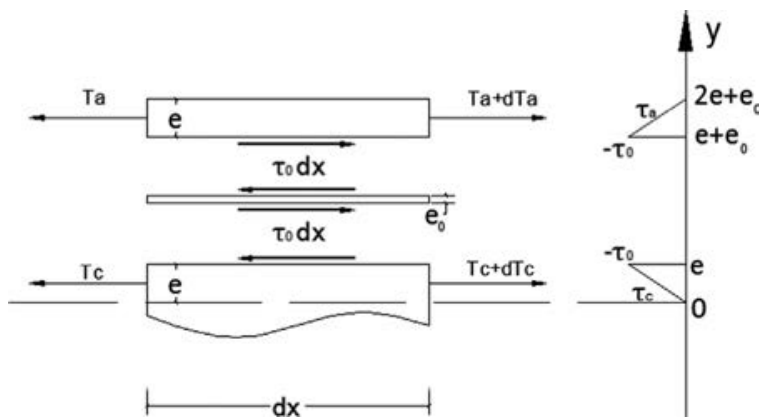

Fig. 2. Assumptions of the improved shear-lag model [16].
The purpose of this work is to calculate the stress wave in the adhesive joint induced by an impulsive and indicial stress wave applied to the middle substrate at the cross-section of $x=0$.

\subsection{Differential equations}

According to shear-lag model, the adhesive shear stress is a linear function of the substrates axial displacements. In the improved shear-lag model, this holds except the shear stress is expressed in terms of the displacements at the adherend-adhesive interfaces [16]. Therefore, the adhesive shear stress reads:

$\tau_{0}(\boldsymbol{x}, \boldsymbol{t})=\frac{\boldsymbol{G}_{0}}{\boldsymbol{e}_{0}}\left(\boldsymbol{u}_{c i}(\boldsymbol{x}, \boldsymbol{t})-\boldsymbol{u}_{a i}(\boldsymbol{x}, \boldsymbol{t})\right)$

where $u_{c i}(x, t)=u_{c}(x, y=e, t)$ and $u_{a i}(x, t)=u_{a}\left(x, y=e+e_{0}, t\right)$ are the displacements at substrate-adhesive interfaces.

The improved shear-lag model assumes that substrates are subjected to both normal and shear stresses. Considering that shear stress vanishes at $y=0$ and $y=2 e+e_{0}$ and assuming continuity of the shear stress at the adherend-adhesive interfaces, the displacements in upper and middle substrates are written [21]:

$\left.\boldsymbol{u}_{\boldsymbol{a}}=\boldsymbol{u}_{\boldsymbol{a} i}+\frac{\boldsymbol{\tau}_{0}}{\boldsymbol{G e}} \frac{\left(\boldsymbol{y}-2 \boldsymbol{e}-\boldsymbol{e}_{0}\right)^{2}-\boldsymbol{e}^{2}}{2}\right)=\boldsymbol{u}_{\boldsymbol{a} i}+\boldsymbol{p}(\boldsymbol{y}) \frac{\boldsymbol{\tau}_{0}}{\boldsymbol{G e}}$

and

$\boldsymbol{u}_{\boldsymbol{c}}=\boldsymbol{u}_{\boldsymbol{c} i}+\frac{\boldsymbol{\tau}_{0}}{\boldsymbol{G e}}\left(\frac{\boldsymbol{e}^{2}-\boldsymbol{y}^{2}}{2}\right)=\boldsymbol{u}_{\boldsymbol{c} i}+\boldsymbol{q}(\boldsymbol{y}) \frac{\boldsymbol{\tau}_{0}}{\mathbf{G e}}$,

where $p(y)=\left(\left(y-2 e-e_{0}\right)^{2}-e^{2}\right) / 2$ and $q(y)=\left(e^{2}-y^{2}\right) / 2$.

As per Challita and Othman [21], using the second Newton's law to include the dynamic effects, and the Hooke's law on each of the adherends, yields

$\boldsymbol{E} \boldsymbol{e}\left(\frac{\partial^{2} \boldsymbol{u}_{\boldsymbol{a} i}}{\partial \boldsymbol{x}^{2}}-\frac{\boldsymbol{e}}{3 \boldsymbol{G}} \frac{\partial^{2} \tau_{0}}{\partial \boldsymbol{x}^{2}}\right)=-\boldsymbol{\tau}_{0}-\frac{\rho \boldsymbol{e}^{2}}{3 \boldsymbol{G}} \frac{\partial^{2} \boldsymbol{\tau}_{0}}{\partial \boldsymbol{t}^{2}}+\boldsymbol{\rho} e \frac{\partial^{2} \boldsymbol{u}_{\boldsymbol{a}}}{\partial \boldsymbol{t}^{2}}$

and

$\boldsymbol{E e}\left(\frac{\partial^{2} \boldsymbol{u}_{\boldsymbol{c} i}}{\partial \boldsymbol{x}^{2}}+\frac{\boldsymbol{e}}{3 \boldsymbol{G}} \frac{\partial^{2} \tau_{0}}{\partial \boldsymbol{x}^{2}}\right)=\boldsymbol{\tau}_{0}+\frac{\rho \boldsymbol{e}^{2}}{3 \boldsymbol{G}} \frac{\partial^{2} \tau_{0}}{\partial \boldsymbol{t}^{2}}+\boldsymbol{\rho} e \frac{\partial^{2} \boldsymbol{u}_{\boldsymbol{c} i}}{\partial \boldsymbol{t}^{2}}$

Eqs. (4) and (5) are equivalently rewritten as

$\frac{\partial^{2}\left(\boldsymbol{u}_{\boldsymbol{a} i}+\boldsymbol{u}_{\boldsymbol{c} i}\right)}{\partial \boldsymbol{x}^{2}}=\frac{\boldsymbol{\rho}}{\boldsymbol{E}} \frac{\partial^{2}\left(\boldsymbol{u}_{\boldsymbol{a} i}+\boldsymbol{u}_{\boldsymbol{c} i}\right)}{\partial \boldsymbol{t}^{2}}$

and

$\left(1+\frac{2 \boldsymbol{e} \boldsymbol{G}_{0}}{3 \boldsymbol{G} \boldsymbol{e}_{0}}\right) \frac{\partial^{2}\left(\boldsymbol{u}_{\boldsymbol{c} i}-\boldsymbol{u}_{a i}\right)}{\partial \boldsymbol{x}^{2}}=\frac{2 \boldsymbol{G}_{0}}{e \boldsymbol{e}_{0} \boldsymbol{E}}\left(\boldsymbol{u}_{c i}-\boldsymbol{u}_{a i}\right)+\frac{\rho}{\boldsymbol{E}}\left(1+\frac{2 \boldsymbol{e} \boldsymbol{G}_{0}}{3 \boldsymbol{G} \boldsymbol{e}_{0}}\right) \frac{\partial^{2}\left(\boldsymbol{u}_{\boldsymbol{c} i}-\boldsymbol{u}_{a i}\right)}{\partial \boldsymbol{t}^{2}}$

Actually, Eq. (6) is obtained by adding Eqs. (4) and (5), whereas Eq. (7) is obtained by subtracting Eq. (4) from Eq. (5). $\tau_{0}$ is eliminated by using Eq. (1).

Eqs. (6) and (7) are two partial differential equations in terms of $\left(u_{c i}-u_{a i}\right)$ and $\left(u_{c i}+u_{a i}\right)$. The two equations are decoupled from each other and can be solved separately. Subsequently, it is possible to find separately $u_{c i}$ and $u_{a i}$. It is worth noticing that the shear stress in the adhesive is proportional to $\left(u_{c i}-u_{a i}\right)$. Therefore, Eq. (7) is also a partial differential equation in terms of $\tau_{0}$, the adhesive shear stress. Indeed, substituting Eq. (1) in Eq. (7) yields

$\left(1+\frac{2 \boldsymbol{e} \boldsymbol{G}_{0}}{3 \boldsymbol{G} \boldsymbol{e}_{0}}\right) \frac{\partial^{2} \tau_{0}}{\partial \boldsymbol{x}^{2}}=\frac{2 \boldsymbol{G}_{0}}{e \boldsymbol{e}_{0} E} \boldsymbol{\tau}_{0}+\frac{\boldsymbol{\rho}}{\boldsymbol{E}}\left(1+\frac{2 \boldsymbol{e} \boldsymbol{G}_{0}}{3 \boldsymbol{G} \boldsymbol{e}_{0}}\right) \frac{\partial^{2} \tau_{0}}{\partial \boldsymbol{t}^{2}}$, 


\subsection{Solution in terms of Laplace transforms}

Challita and Othman [21] used complex exponential representation to solve Eqs. (6) to (8). This was suitable for calculating the harmonic response. As we are interested here in impulsive and indicial response, the Laplace transforms are more appropriate [9].

Let $c=\sqrt{E / \rho}$ and $\eta=s / c$ where $s$ is the Laplace parameter. Applying the Laplace transform on Eq. (6) leads to

$\frac{\partial^{2}\left(\boldsymbol{U}_{\boldsymbol{a} i}+\boldsymbol{U}_{\boldsymbol{c} i}\right)}{\partial \boldsymbol{x}^{2}}-\boldsymbol{\eta}^{2}\left(\boldsymbol{U}_{\boldsymbol{a} i}+\boldsymbol{U}_{\boldsymbol{c} i}\right)=0$

where $U_{a i}(x, s)$ and $U_{c i}(x, s)$ are the Laplace transforms of $u_{a i}(x, t)$ and $u_{c i}(x, t)$, respectively. Actually, the establishment of Eq. (9) assumes that the adhesive joint is at rest at $(t=0)$; hence $\partial u_{a i} / \partial t(x, 0)=\partial u_{c i} / \partial t(x, 0)=0$ and $u_{a i}(x, 0)=u_{c i}(x, 0)=0$.

Now, let $\mu=\sqrt{G_{0} / e e_{0} E}, \quad \theta=\sqrt{1+\left(2 e G_{0} / 3 e_{0} G\right)}$ and $\lambda=\sqrt{\eta^{2}+2\left(\mu^{2} / \theta^{2}\right)}$.

Using the Laplace transform for Eq. (7) yields

$\frac{\partial^{2}\left(\boldsymbol{U}_{\boldsymbol{c} \boldsymbol{i}}-\boldsymbol{U}_{\boldsymbol{a} i}\right)}{\partial \boldsymbol{x}^{2}}-\lambda^{2}\left(\boldsymbol{U}_{\boldsymbol{c} \boldsymbol{i}}-\boldsymbol{U}_{\boldsymbol{a} i}\right)=0$

$\eta$ and $\lambda$ are two constants of unit $\left[\mathrm{m}^{-1}\right]$. $\lambda$ appears later in Eq. (35), where it describes the coefficient of exponential decrease of the adhesive shear stress along overlap length. Similarly, $\eta$ is the exponential coefficient that describes damping of the normal stress in the substrates.

The solutions of Eqs. (9) and (10) are expressed in terms of hyperbolic trigonometric functions as follows:

$\boldsymbol{U}_{\boldsymbol{a} i}+\boldsymbol{U}_{\boldsymbol{c} \boldsymbol{i}}=\boldsymbol{A} \cosh (\boldsymbol{\eta} x)+\boldsymbol{B} \sinh (\boldsymbol{\eta} x)$

and

$\boldsymbol{U}_{\boldsymbol{c} \boldsymbol{i}}-\boldsymbol{U}_{\boldsymbol{a} \boldsymbol{i}}=\boldsymbol{C} \cosh (\lambda x)+\boldsymbol{D} \sinh (\boldsymbol{\lambda} x)$,

respectively. $A, B, C$ and $D$ are constants that can depend on $s$ and will be determined considering boundary conditions at $x=0$ and $x=\infty$. Subtracting Eq. (12) from Eq. (11) leads to

$\boldsymbol{U}_{\boldsymbol{a} \boldsymbol{i}}=\frac{1}{2}(\boldsymbol{A} \cosh (\boldsymbol{\eta} x)+\boldsymbol{B} \sinh (\boldsymbol{\eta} x)-\boldsymbol{C} \cosh (\boldsymbol{\lambda} x)-\boldsymbol{D} \sinh (\lambda x))$

On the other hand, adding Eqs. (11) to (12) yields

$\boldsymbol{U}_{\boldsymbol{c} \boldsymbol{i}}=\frac{1}{2}(\boldsymbol{A} \cosh (\boldsymbol{\eta} x)+\boldsymbol{B} \sinh (\boldsymbol{\eta} x)+\boldsymbol{C} \cosh (\boldsymbol{\lambda} x)+\boldsymbol{D} \sinh (\boldsymbol{\lambda} x))$

The Laplace transform of the adhesive shear stress, $T_{0}(x, s)$, is deduced by applying Laplace transform to Eq. (1) and replacing the displacements at adherend-adhesive interfaces by their solutions in Eqs. (13) and (14). Namely,

$\boldsymbol{T}_{0}(\boldsymbol{x}, \boldsymbol{s})=\frac{\boldsymbol{G}_{0}}{\boldsymbol{e}_{0}}(\boldsymbol{C} \cosh (\lambda x)+\boldsymbol{D} \sinh (\lambda x))$

The Laplace transform of the substrates axial displacement is deduced by substituting Eqs. (13) and (14) in the Laplace transforms of Eqs. (2) and (3), respectively. Namely,

$$
\begin{aligned}
& \boldsymbol{U}_{\boldsymbol{a}}(\boldsymbol{x}, \boldsymbol{y}, \boldsymbol{s})=\frac{\boldsymbol{A}}{2} \cosh (\boldsymbol{\eta} x)+\frac{\boldsymbol{B}}{2} \sinh (\boldsymbol{\eta} x) \\
& \quad+\boldsymbol{C}\left(\frac{\boldsymbol{G}_{0} \boldsymbol{p}(\boldsymbol{y})}{\boldsymbol{G e}_{0}}-\frac{1}{2}\right) \cosh (\lambda x)+\boldsymbol{D}\left(\frac{\boldsymbol{G}_{0} \boldsymbol{p}(\boldsymbol{y})}{\boldsymbol{G e e}_{0}}-\frac{1}{2}\right) \sinh (\lambda x),
\end{aligned}
$$

and

$$
\begin{aligned}
\boldsymbol{U}_{\boldsymbol{c}}(\boldsymbol{x}, \boldsymbol{y}, \boldsymbol{s})= & \frac{\boldsymbol{A}}{2} \cosh (\boldsymbol{\eta} x)+\frac{\boldsymbol{B}}{2} \sinh (\boldsymbol{\eta} x)+\boldsymbol{C}\left(\frac{\boldsymbol{G}_{0} \boldsymbol{q}(\boldsymbol{y})}{\boldsymbol{G e}_{0}}+\frac{1}{2}\right) \cosh (\lambda x) \\
& +\boldsymbol{D}\left(\frac{\boldsymbol{G}_{0} \boldsymbol{q}(\boldsymbol{y})}{\boldsymbol{G e}_{0}}+\frac{1}{2}\right) \sinh (\lambda x)
\end{aligned}
$$

We would like to attract the attention of the reader that Eqs. (13) and (14) are similar to Eq. (17) of Ref. [9], except that the coefficient $\lambda$ is equal to $\sqrt{\eta^{2}+2 \mu^{2}}$ in [9] and not $\sqrt{\eta^{2}+2\left(\mu^{2} / \theta^{2}\right)}$ as in this work. Actually the two expressions of $\lambda$ converge if the shear stiffness of the adhesive $G_{0} / e_{0}$ is insignificant compared to shear stiffness of the substrates $G / e$. Indeed, $\theta \approx 1$ if $G_{0} / e_{0} \ll G / e$.

In other words, the model developed in this work converges to Sato's model if the substrates are highly shear-stiff compared to the adhesive. Moreover, the two models take into account inertia effects in the same way. On one hand, adhesive inertia is neglected. On the other hand, only longitudinal inertia of substrates is considered by the two models. Thus, any difference in results predicted by the two models should be attributed to the shear stiffness ratio and not to inertia.

\subsection{Boundary conditions}

As a boundary condition, a stress wave was assumed in the virtual interface of the middle substrate at the edge of the joint or at $x=0$. Evidently, no reflections occur at the infinities.

The solutions obtained in Section 2.3 are expressed in terms of four s-dependent functions $A, B, C$ and $D$. Therefore, four boundary conditions are needed to obtain the final solution. Actually, it is possible to write two boundary equations, at $x=0$ and $x=\infty$, for each of the two substrates.

The two boundary conditions at $(x=0)$ are more complex to establish for this improved shear-lag model than for the simple shear-lag model because the shear stress is not neglected in the substrates.

Let us start with the boundary condition at $x=0$ for the central substrate (Fig. 3). Actually, the incident rightwards wave, $\sigma_{i}(x<0, t)$, propagating in the left part of the middle substrate, is a pressure wave. Hence, the axial stress is constant through the thickness and the shear stress is absent for $(x<0)$. On the opposite, shear and normal stresses coexist in the right part $(x>0)$ of the middle substrate. Regarding these observations, the improved shear-lag model cannot take into account the continuity of stress state at $(x=0)$. However, we can write second Newton's law on a small slice of the middle substrate around $(x=0)$, more precisely for $(-\xi \leq x \leq \xi)$. This leads to:

$-\boldsymbol{T}_{\boldsymbol{c}}(-\boldsymbol{\xi}, \boldsymbol{t})+\boldsymbol{T}_{\boldsymbol{c}}(\boldsymbol{\xi}, \boldsymbol{t})-\boldsymbol{l} \int_{0}^{+\boldsymbol{\xi}} \tau \mathrm{dx}=\boldsymbol{l} \int_{0}^{\boldsymbol{e}} \int_{-\boldsymbol{\xi}}^{+\boldsymbol{\xi}} \boldsymbol{\rho} \frac{\partial^{2} \boldsymbol{u}_{\boldsymbol{a}}}{\partial \boldsymbol{t}^{2}} \mathrm{dx} \mathrm{dy}$

where $\xi$ is a real constant, $l$ is the width the adhesive joint. We also recall that $T_{a}$ is the axial normal force. When $\xi$ approaches 0 , Eq. (18) converges to:

$-\boldsymbol{T}_{c}\left(0^{-}, \boldsymbol{t}\right)+\boldsymbol{T}_{c}\left(0^{+}, \boldsymbol{t}\right)=0$

Even though, the improved shear-lag model cannot assure the continuity of stress state at $x=0$, it can guarantee axial force continuity.

At $x=0$, the axial normal force from the left side is simply:

$\boldsymbol{T}_{\boldsymbol{c}}\left(0^{-}, \boldsymbol{t}\right)=e l \boldsymbol{\sigma}_{\boldsymbol{i}}\left(0^{-}, \boldsymbol{t}\right)$

because the axial stress of the incident wave is assumed constant through the cross-section of the central substrate. On the other

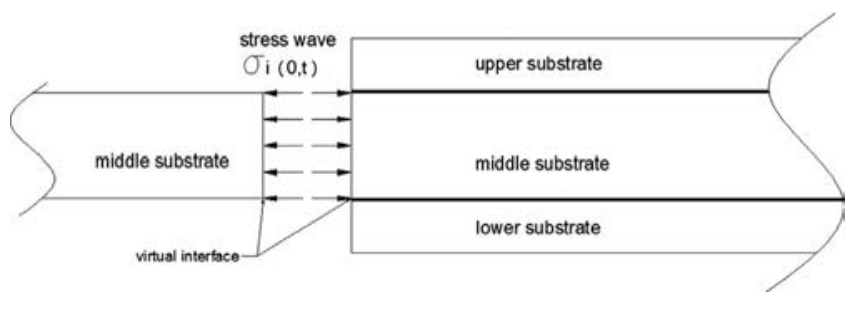

Fig. 3. Loading condition. 
hand, the axial stress from the right side is given by

$\boldsymbol{T}_{\boldsymbol{c}}\left(0^{+}, \boldsymbol{t}\right)=\boldsymbol{l} \int_{0}^{\boldsymbol{e}} E \frac{\partial \boldsymbol{u}_{\boldsymbol{c}}}{\partial \boldsymbol{x}}\left(0^{+}, \boldsymbol{y}, \boldsymbol{t}\right) \mathrm{dy}$.

Substituting Eqs. (20) and (21) in Eq. (18) and using Laplace transform leads to:

$\int_{0}^{\boldsymbol{e}} E \frac{\partial \boldsymbol{U}_{\boldsymbol{c}}}{\partial \boldsymbol{x}}\left(0^{+}, \boldsymbol{y}, \boldsymbol{s}\right) \mathrm{dy}=e \boldsymbol{\Sigma}_{\boldsymbol{i}}\left(0^{-}, \boldsymbol{s}\right)$

wherein $\Sigma_{i}$ holds for the Laplace transform of the incident wave $\sigma_{i}$.

The left edge of the upper substrate is assumed free. However, the improved shear-lag model cannot consider the stress is zero at $(x=0)$. The approach used to establish Eqs. (18-22) can be applied to the upper substrate. Thus we can write that the axial force vanishes at $(x=0)$ :

$\boldsymbol{T}_{\boldsymbol{a}}\left(0^{+}, \boldsymbol{t}\right)=0$.

or in terms of Laplace transform:

$\int_{0}^{\boldsymbol{e}} \frac{\partial \boldsymbol{U}_{\boldsymbol{a}}}{\partial \boldsymbol{x}}\left(0^{+}, \boldsymbol{y}, \boldsymbol{s}\right) \mathrm{dy}=0$.

Eqs. (23) and (24) give the first and second boundary conditions. Substituting Eq. (17) in Eq. (22) and calculating the integral yields:

$\frac{\eta}{2} B+\frac{\lambda \theta^{2}}{2} D=\frac{\Sigma_{i}\left(0^{-}, s\right)}{E}$

Likewise, Substituting Eq. (16) in Eq. (24) and calculating the integral:

$\frac{\boldsymbol{\eta}}{2} \boldsymbol{B}-\frac{\lambda \boldsymbol{\theta}^{2}}{2} \mathrm{D}=0$

These two last equations gave the weighted sum and difference of $B$ and $D$. Their solution is straightforward and reads:

$\boldsymbol{B}=\frac{\Sigma_{i}\left(0^{-}, s\right)}{\eta E}$

and

$\boldsymbol{D}=\frac{\Sigma_{\boldsymbol{i}}\left(0^{-}, \boldsymbol{s}\right)}{E \lambda \boldsymbol{\theta}^{2}}$.

In order to get now $A$ and $C$, we need two more boundary equations, namely at $x=\infty$. The adhesive joint is assumed semiinfinite. Within a finite time duration, it is impossible to the stress wave to reach infinity. Thus, the substrates' displacement should vanish at $x=\infty$. In other words,

$\boldsymbol{u}_{c}(\boldsymbol{x}=\infty, \boldsymbol{y}, \boldsymbol{t})=\boldsymbol{u}_{\boldsymbol{a}}(\boldsymbol{x}=\infty, \boldsymbol{y}, \boldsymbol{t})=0$

In terms of Laplace transform, these boundary conditions are written:

$\boldsymbol{U}_{\boldsymbol{c}}(\boldsymbol{x}=\infty, \boldsymbol{y}, \boldsymbol{s})=\boldsymbol{U}_{\boldsymbol{a}}(\boldsymbol{x}=\infty, \boldsymbol{y}, \boldsymbol{s})=0$

Considering $\sinh (\infty) \approx \cosh (\infty)$ and substituting Eqs. (16) and (17) in Eqs. (29) and (30) leads to

$\frac{\boldsymbol{A}}{2}+\frac{\boldsymbol{B}}{2}+\boldsymbol{C}\left(\frac{\boldsymbol{G}_{0} \boldsymbol{p}(\boldsymbol{y})}{\boldsymbol{G e e}_{0}}-\frac{1}{2}\right)+\boldsymbol{D}\left(\frac{\boldsymbol{G}_{0} \boldsymbol{p}(\boldsymbol{y})}{\boldsymbol{G e e}_{0}}-\frac{1}{2}\right)=0$

and

$\frac{\boldsymbol{A}}{2}+\frac{\boldsymbol{B}}{2}+\boldsymbol{C}\left(\frac{\boldsymbol{G}_{0} \boldsymbol{q}(\boldsymbol{y})}{\boldsymbol{G e e}_{0}}+\frac{1}{2}\right)+\boldsymbol{D}\left(\frac{\boldsymbol{G}_{0} \boldsymbol{q}(\boldsymbol{y})}{\boldsymbol{G e e}_{0}}+\frac{1}{2}\right)=0$

This is only possible if:

$\boldsymbol{A}=-\boldsymbol{B}$

and

$\boldsymbol{C}=-\boldsymbol{D}$
Finally, substituting Eqs. (28) and (34) in Eq. (15) yields

$\boldsymbol{T}_{0}(\boldsymbol{x}, \boldsymbol{s})=-\frac{\boldsymbol{G}_{0}}{\boldsymbol{e}_{0}} \frac{\boldsymbol{\Sigma}_{\boldsymbol{i}}\left(0^{-}, \boldsymbol{s}\right)}{E \lambda \boldsymbol{\theta}^{2}} \exp (-\lambda x)=\boldsymbol{T}_{0}(0, \boldsymbol{s}) \exp (-\lambda x)$

We recall that $T_{0}(x, s)$ is the Laplace transform of, $\tau_{0}(x, t)$, the shear stress in the adhesive layer. It is worth noticing here that Eq. (35) gives that the adhesive shear stress vanishes at infinity.

\subsection{Transfer function between incident stress and edge adhesive} shear stress

The main goal of this paper is to calculate the stress response of the double-lap joint to impulse and indicial stress wave. To achieve this aim, the double-lap joint can be regarded as a system for which the transfer function is the ratio of the edge shear stress in the adhesive joint divided by the incident stress wave. The edge shear stress is the shear stress at $x=0^{+}$. The transfer function, $H$, is defined as

$\boldsymbol{H}(\boldsymbol{s})=\frac{\boldsymbol{T}_{0}\left(0^{+}, \boldsymbol{s}\right)}{\boldsymbol{\Sigma}_{\boldsymbol{i}}\left(0^{-}, \boldsymbol{s}\right)}$

Considering $x=0^{+}$in Eq. (35) and replacing in Eq. (36) leads to:

$\boldsymbol{H}(\boldsymbol{s})=-\frac{\boldsymbol{G}_{0}}{\boldsymbol{e}_{0} E \lambda \boldsymbol{\theta}^{2}}=-\frac{c \boldsymbol{G}_{0}}{\boldsymbol{e}_{0} E \boldsymbol{\theta}^{2}} \frac{1}{\sqrt{\boldsymbol{s}^{2}+\frac{2 \mu^{2} \boldsymbol{c}^{2}}{\boldsymbol{\theta}^{2}}}}$

Here also we notice if $\theta$ approaches to 1 , which is possible if the shear stiffness of the adhesive is insignificant compared to the shear stiffness of the substrates, Eq. (37) converges to Eq. (21) of Ref. [9].

\subsection{Impulse and indicial responses}

The impulse response is defined as the response of a system subjected to an impulse loading, represented by the Dirac's Delta; it can be brought by the inverse-Laplace of the transfer function given in Eq. (37). Knowing that the inverse Laplace transform of $G(s)=1 / \sqrt{s^{2}+\omega^{2}}$ is the function $g(t)=u(t) \times J_{0}(\omega t)$, wherein $u()$ and $J_{0}()$ denote the Heaviside unit step function and the zero-order Bessel function respectively; the inverse-Laplace transform of the transfer function is:

$\left.\boldsymbol{\tau}_{0}^{i m p}(0, \boldsymbol{t})=\boldsymbol{L}^{-1}(\boldsymbol{H}(\boldsymbol{s}))=\frac{-\boldsymbol{c} G_{0}}{\boldsymbol{e}_{0} E \boldsymbol{\theta}^{2}} J_{0} \frac{\sqrt{2} \boldsymbol{\mu c}}{\boldsymbol{\theta}} \boldsymbol{t}\right) \boldsymbol{u}(\boldsymbol{t})$

where $\tau_{0}^{i m p}(0, t)$ is the shear stress at the edge of the adhesive layer due to an impulse load.

The indicial response is the response of a system subjected to the Heaviside step function; it can be considered as the response to an infinite sum of delayed impulse responses. This is mathematically written as a convolution product. More precisely, the indicial response $\tau_{0}^{\text {ind }}(0, t)$ is the convolution product between the impulse response $\tau_{0}^{i m p}(0, t)$ and the Heaviside unit step function $u(t)$ :

$\boldsymbol{\tau}_{0}^{i n d}(0, t)=\boldsymbol{\tau}_{0}^{i m p}(0, t) * u(t)$

Hence, the indicial response is obtained by calculating the integral of the impulse response on the interval $[0, t]$, i.e.,

$\boldsymbol{\tau}_{0}^{\boldsymbol{i n d}}(0, \boldsymbol{t})=\frac{-\boldsymbol{c} \boldsymbol{G}_{0}}{\boldsymbol{e}_{0} E \boldsymbol{\theta}^{2}} \int_{0}^{\boldsymbol{t}} J_{0}\left(\frac{\sqrt{2} \boldsymbol{\mu} c}{\boldsymbol{\theta}} \boldsymbol{t}^{\prime}\right) \mathrm{dt}^{\prime}$

It is worth noting that the indicial response converges to a finite value for infinite time. Indeed,

$\lim _{\boldsymbol{t} \rightarrow+\infty} \boldsymbol{\tau}_{0}^{\text {ind }}(0, \boldsymbol{t})=\lim _{\boldsymbol{s} \rightarrow 0} \boldsymbol{H}(\boldsymbol{s})=-\frac{1}{\boldsymbol{\theta}} \sqrt{\frac{\boldsymbol{G}_{0} \boldsymbol{e}}{2 \boldsymbol{e}_{0} \boldsymbol{E}}}$ 
Assuming that $\theta$ tends to 1 , which means that the substrates' shear stiffness is much higher than that of the adhesive layer, Eq. (41) converges to $-\sqrt{e G_{0} / 2 E e_{0}}$ which recalls Eq. (29) from Ref. [9]. On the other hand, the value in Eq. (41) is exactly equal to the value found if the infinite length joint is subjected to static unitary loading as modelled by the quasi-static improved shear-lag model developed by Tsai et al. [16]. In other words, the dynamic shear stress in the adhesive layer converges, after long period of time, to the static shear stress.

\section{Validation and discussion}

In this section, we aim at validating the improved shear-lag model as extended in this work to predict impulsive and indicial response for a double lap bounded joint. First, we define the following constants: $\varphi_{\text {Sato }}=\sqrt{e G_{0} / 2 E e_{0}}$ and $\varphi=\varphi_{\text {Sato }} / \theta$, which stand for the absolute value of the indicial response after infinite time as obtained by simple shear-lag [9] and the improved shearlag (current work), respectively. We also define the constant $\Psi=G_{0} c / E e_{0} \theta^{2}$. These three constants are used in order to depict dimensionless results. In what follows, the improved shear-lag model is compared to the simple shear-lag model and finite element method. The finite element model is undertaken with Abaqus software. It assumes an adhesive shear modulus of $0.71 \mathrm{GPa}$, thickness of $0.2 \mathrm{~mm}$ and adherends thickness $(e)$ of $2 \mathrm{~mm}$. Physically, the infinite length of the joint was presented in the finite element model in a way to prevent the reflected wave from arriving back to $x=0$ during the time of simulation which is taken equal to $500 \mu \mathrm{s}$.

\subsection{Effect of substrates' material}

\subsubsection{Steel substrates}

First, we investigated the impulse and indicial response of a double-lap joint made of steel substrates. Indeed, steel alloys have a Young's modulus about $200 \mathrm{GPa}$ and a shear modulus around $77 \mathrm{GPa}$. Thus, the ratio of the adhesive layer's shear stiffness to the substrates' shear stiffness is very small. Therefore, the improved shear-lag model should converge to the Sato's model. Actually, the constant $\theta \approx 1.03$. Figs. 4 and 5 show the dimensionless impulse and indicial responses, respectively. The two models show a good agreement. The relative difference between the successive maxima of oscillations does not exceed $6 \%\left(\approx \theta^{2}-1\right)$. It is unnecessary to compare to finite element simulation as Sato's model was validated numerically for metallic thin adherends. Consequently our model is valid as well for the same substrates.

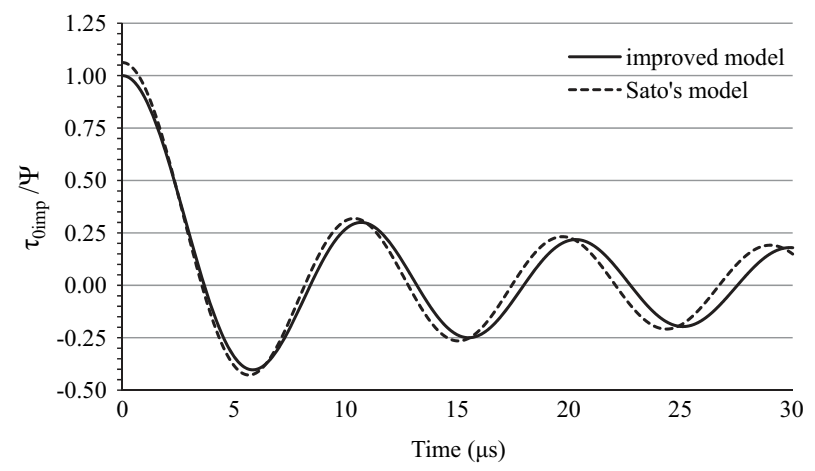

Fig. 4. Dimensionless impulse responses for steel adherends.

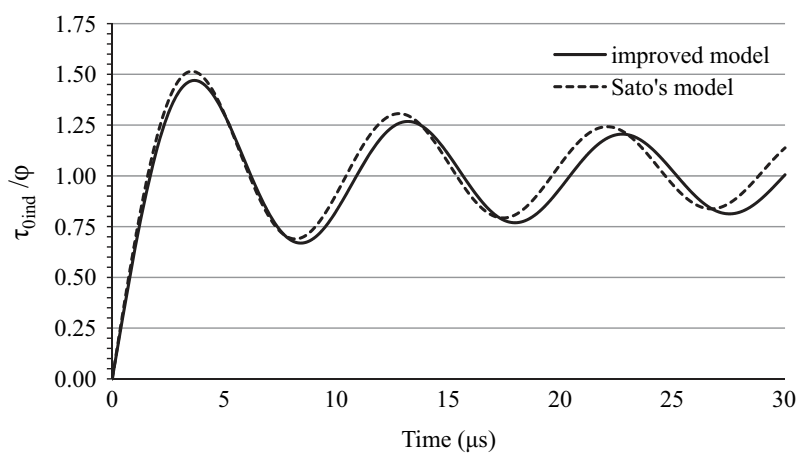

Fig. 5. Dimensionless indicial responses for steel adherends.

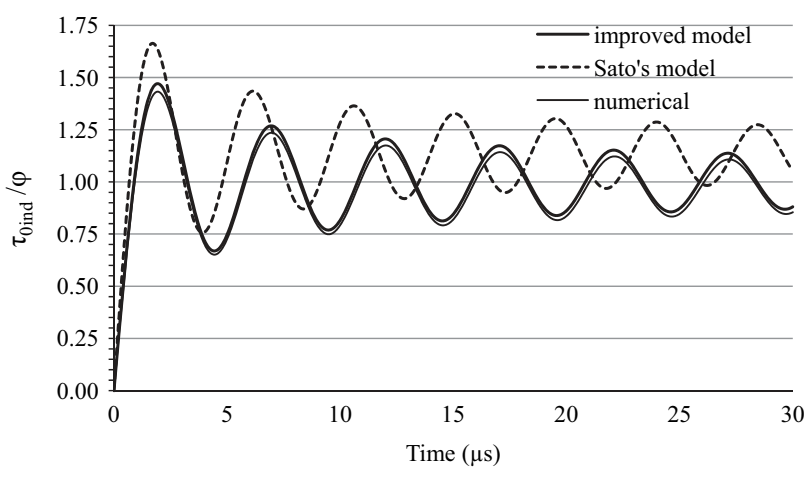

Fig. 6. Dimensionless indicial responses for magnesium adherends.

\subsubsection{Magnesium substrates}

The previous study, related steel substrates, showed a good agreement between the two models. One cannot conclude which one of the models gives better results. It is of much importance to choose a configuration where the two models are expected to give different results. To this aim, we have studied a double-lap adhesive joint made of magnesium substrates. Actually magnesium has a lower Young's modulus (shear modulus) compared to the steel. The magnesium's Young's modulus is of $45 \mathrm{GPa}$ and the shear modulus of $17 \mathrm{GPa}$. In this case the constant $\theta \approx 1.13$.

Fig. 6 shows the dimensionless indicial response for the three models. Whereas, the new improved shear-lag response agrees well with the finite element response, while the simple shear lagmodel, of Ref. [9], has some difficulties to catch the numericallypredicted response. More precisely, Sato's model [9] overestimates the maxima of oscillations while underestimating the period of oscillations. This is mainly due to $\theta>1$. Moreover, the stress damping in terms of time is more important for Sato's model than the current, this is caused by the fact that the period of oscillations is lower for the former model. However, the simple shear-lag model converges, after infinite time, to a shear stress value higher than the predicted by the finite element and the current improved shear-lag models.

Fig. 7 plots the dimensionless impulse response predicted by the two analytical methods. Here also Sato's model [9] overestimates the maxima of oscillations and underestimates the period of oscillations. The relative difference of maxima predicted is around $25 \%$ which is approximately equal to $\left(\theta^{2}-1\right)$.

\subsubsection{Polymer substrates}

In the previous sections, we have shown that our improved shear-lag model predicts approximately the same response as the finite element model, when metallic substrates were involved. In this section, we were interested in validating our model for 


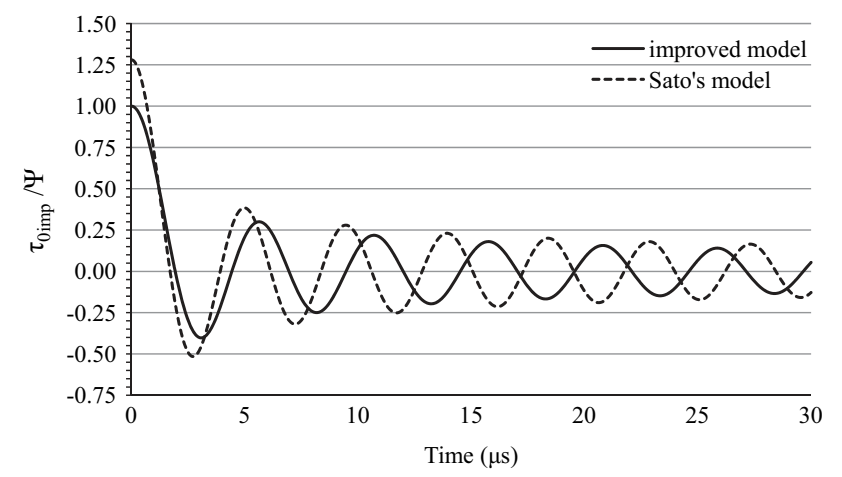

Fig. 7. Dimensionless impulse responses for magnesium adherends.

polymer-like materials. In this case, the shear modulus of the substrates is of the same order as the shear modulus of the adhesive. For this purpose, a finite element simulation was carried out using isotropic polymer material properties for both adherends and adhesive. The adherends' Young's modulus was considered equal to $4 \mathrm{GPa}$ and shear modulus of $1.42 \mathrm{GPa}$. In this case, $\theta \approx 2.08$. The dimensionless indicial response predicted by the three models (simple shear-lag, improved shear-lag and finite element) are depicted in Fig. 8. The new model agrees well with the finite element analysis. It catches with good accuracy the maxima and period of oscillations. The maximum error is less than $12 \%$. It is worth noting that the two responses are in-phase, with almost no time shift. On the opposite, Sato's model highly overestimates the shear stress in the adhesive and underestimates the period of oscillation. This could be explained by the significant value of the shear adhesive-to-adherend stiffness ratio, which is of order of 5 .

\subsubsection{Composite substrates}

In the above three examples, metallic and polymer-like materials were considered. The material behaviour was isotropic. In this section, we are interested in validating the improved shear-lag model against an orthotropic material. For this purpose, we assumed substrates made of an orthotropic composite unidirectional laminates. For instance, we choose adherands made of APC2/AS4 PEEK reinforced with 60\% carbon fibre laminates. Thus substrates have a longitudinal Young's modulus of $138 \mathrm{GPa}$, transverse Young's modulus of $10.2 \mathrm{GPa}$, in-plane shear modulus of $5.7 \mathrm{GPa}$ and a density of $1.5 \mathrm{~g} / \mathrm{cc}$. [22].

Fig. 9 shows the indicial response calculated by the three approaches, i.e., the improved shear-lag model, Sato's model and the finite element method. It is shown that the new model, developed in this paper, overcomes Sato's model. It is worth noting here that what controls the accuracy of Sato's model is the shear adherends-to-adhesive stiffness ratio. Indeed, the longitudinal Young's modulus of substrates is $138 \mathrm{GPa}$, which is much higher that the shear stiffness of the adhesive. However, the Sato's model fails to estimate the shear stress in the adhesive because the in-plane shear modulus of the adhesive $(\sim 5.7 \mathrm{GPa})$ is comparable to the shear modulus of the adhesive ( $3 \mathrm{GPa}$ ).

3.1.4.1. Effect of substrates' thickness (e). In the previous sections, we compared our model to Sato's model for different substrates' materials or for different values of substrates' shear modulus. In this section, we aimed to show that the most important parameter in the double-lap response is shear stiffness ratio and not the shear modulus ratio. More precisely, we compared here different double-lap joints having different substrates' materials while

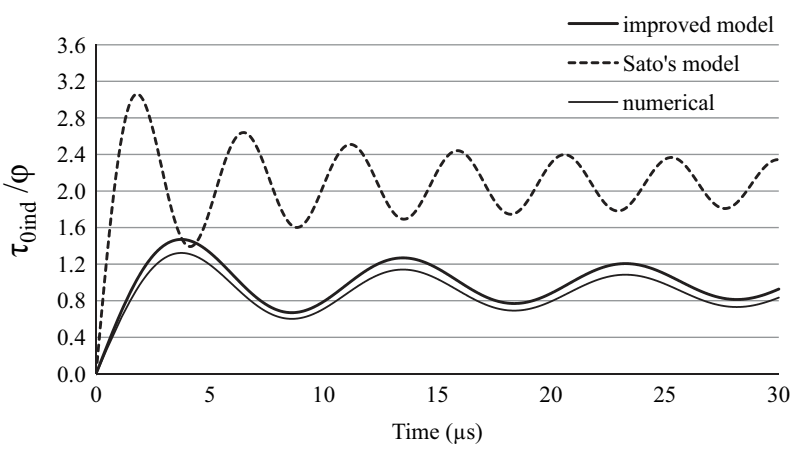

Fig. 8. Dimensionless indicial responses for polymer adherends.

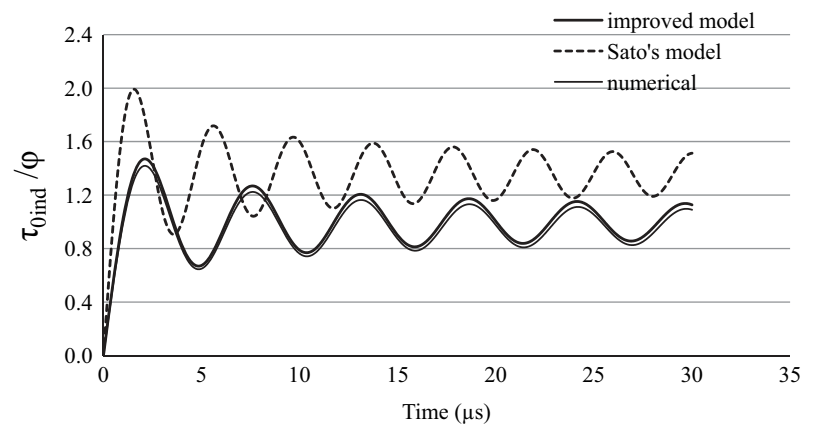

Fig. 9. Dimensionless indicial responses for high Young's modulus UD composite adherends.

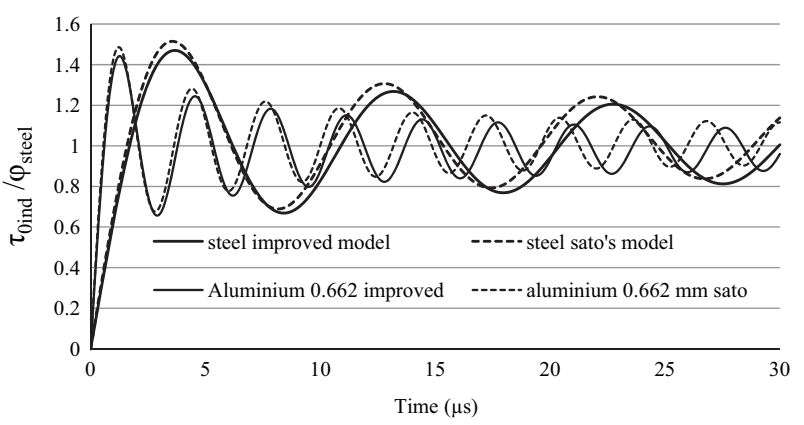

Fig. 10. Dimensionless indicial responses for steel and aluminium double lapjoints.

maintaining the shear substrate-to-adhesive stiffness ratio constant.

As a first example, we compared our results to Sato's results for Steel adherends of $2 \mathrm{~mm}$ thickness and aluminium adherends of $0.662 \mathrm{~mm}$ thickness with a shear modulus of $25.5 \mathrm{GPa}$ (Fig. 10). In this case, the steel and aluminium double-lap joints have the same shear stiffness ratios of approximately 0.01 . Fig. 10 shows that both models are in agreement.

As a second example, we considered the magnesium doublelap joint where substrates are 2 mm-thick together with an aluminium double-lap joint where substrates are $3 \mathrm{~mm}$-thick. The indicial responses of the two double-lap joints are shown in Fig. 11. Clearly, the response predicted by Sato's model is distant from that given by the improved shear-lag model.

The curves of Figs. 4-11 appear like if there is some damping or energy dissipation in the model, yet, the model is perfectly elastic and no material damping was considered. Actually, the impulsive and indicial input loads have a large spectrums. Therefore, the initial energy is distributed on an infinite number of harmonic components which are synchronized in the beginning in a way to 


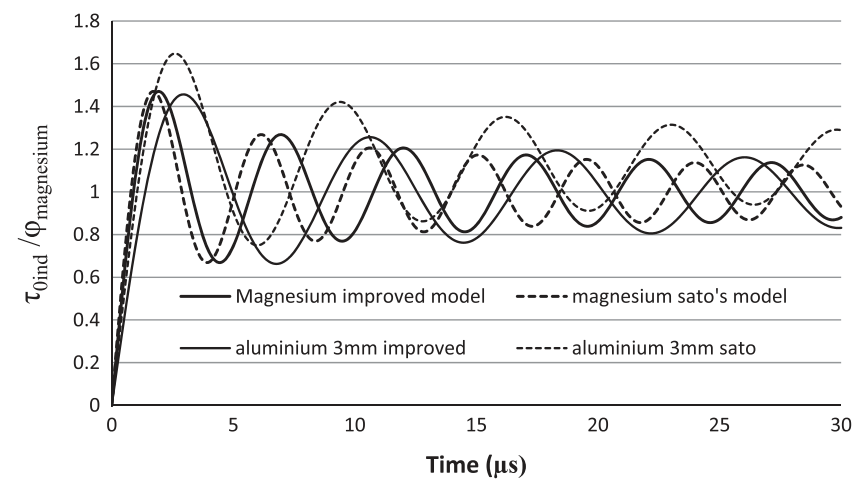

Fig. 11. Dimensionless indicial responses for magnesium and aluminium doublelap joints.

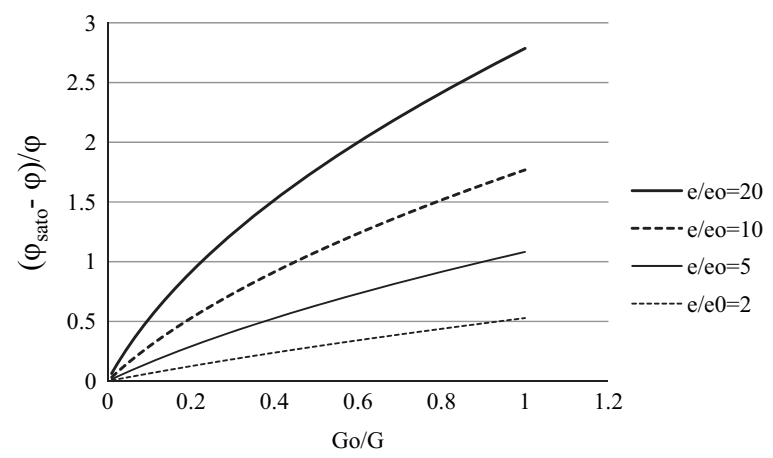

Fig. 12. Sato's model relative error to the improved model for estimating the convergence value when time tends to infinity for a wide range of shear stiffness ratio.

give the impulsive or the indicial shape. However, single harmonic components differently transfer, from the adherend to the adhesive joint. The transfer function should depend on the frequency indeed. A shift is then created between single harmonic waves which results in the new Bessel shape. In other words, the decrease in amplitude is due to the a-synchronization of the harmonic components of the load while they transfer from the middle adherend to the adhesive.

It is also worth noting that in Figs. 6, 8, 9 and 11, Sato's model predicts higher shear stresses in the adhesive and lower period of oscillation in terms of the indicial response. Actually, Sato's model only accounts for normal stresses in the adherends, whereas the improved shear-lag model, proposed here, accounts for both normal and shear stresses. Therefore, Sato's model results depict the effects of substrates normal deformation on the adhesive shear stress. The difference between Sato's model and the new proposed model depicts the effects of the substrates shear deformation on the adhesive shear stress.

\subsection{Validity of Sato's model}

From the discussion made in Sections 3.1 and 3.2, we can conclude that the accuracy of Sato's model highly depends on the shear stiffness ratio. Considering the new improved shear lag model, developed in this paper, as reference, we can estimate the relative error of Sato's model. More precisely, we are interested in the indicial response after infinite time and the period of oscillation. Hence, we recall $\varphi_{\text {Sato }}=\sqrt{e G_{0} / 2 E e_{0}}$ and $\varphi=\varphi_{\text {Sato }} / \theta$, which stand for the absolute value of the indicial response after infinite time as obtained by simple and improved shear-lag. We also define the period of oscillation for the improved model per $p=\theta / \sqrt{2} \mu c$ and for Sato's model per $p_{\text {sato }}=1 / \sqrt{2} \mu c$.

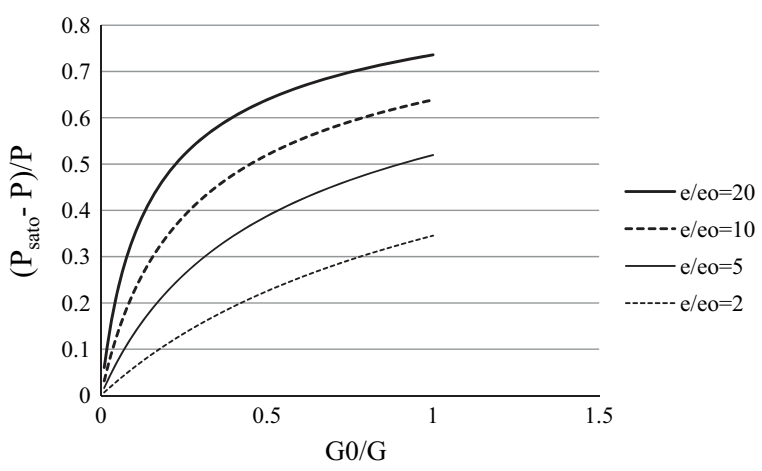

Fig. 13. Sato's model relative error to the improved model for estimating the period of oscillations for a wide range of shear stiffness ratio.

Fig. 12 shows Sato's model relative error to the improved model for estimating the convergence value when time tends to infinity in terms of the shear modulus ratio for four values of thickness ratio. Likewise, Fig. 13 shows the relative error for the period of oscillation in terms of the shear modulus ratio for four values of thickness ratio. As expected, the two errors increase with increasing $G_{0} / G$ and $e / e_{0}$.

\section{Conclusion}

An analytical model was developed for the dynamic response of half-infinite length double lap polymer joint subjected to impact loadings assuming no reflections at the adhesive edge. The work, based on the improved shear-lag model, consisted in taking into consideration the shear deformation inside adherends while neglecting adhesive inertia. Adhesive shear stress was deduced from adherends-adhesive interfaces relative displacements. These displacements were extracted from ordinary differential equations obtained from the equilibrium equations of the joint. Adherends displacements were used along with normal forces to apply boundary conditions. Laplace transform was used to solve the differential equations. Considering the joint as a system, the transfer function between applied load and adhesive edge shear stress was extracted. The joint adhesive edge shear stress impulse response was extracted using the inverse Laplace's transform of the transfer function. Impulse response appeared to be a zero-order Bessel function. Indicial response can be brought by integrating the impulse response. Theoretically, the system showed convergence to the static value when time tends to infinity. A term appeared in the response indicating adhesive to adherend shear stiffness ratio. This term can be neglected for thin shear-stiff adherends, therefore, the system response has shown very good agreement with Sato's model which is based on the pioneer work of Volkersen. The new model was validated regarding finite element results for different substrates materials and different values of the shear substrate-to-adhesive stiffness ratios.

\section{References}

[1] Challita G, Othman R, Guégan P, Khalil K, Poitou A. New experimental sample for shear testing of adhesively bonded assemblies. Int J Mod Phys B 2008;22:1081-6.

[2] Challita G, Othman R, Lebrun JM, Casari P, Guégan P. Determination of dynamic shear stress strain relationship in adhesively bonded assemblies. Proc DYMAT 2009;1:275-80. http://dx.doi.org/10.1051/dymat/2009038.

[3] Challita G, Othman R, Casari P, Khalil K. Experimental investigation of the shear dynamic behavior of double-lap adhesively bonded joints on a wide range of strain rates. Int J Adhes Adhes 2011;31:146-53.

[4] Al-Zubaidy HA, Zhao XL, Al-Mahaidi R. Experimental evaluation of the dynamic bond strength between CFRP sheets and steel under direct tensile loads. Int J Adhes Adhes 2013;40:89-102. 
[5] Challita G, Othman R. Finite-element analysis of SHPB tests on double-lap adhesive joints. Int J Adhes Adhes 2010;30:236-44.

[6] Hazimeh R, Challita G, Khalil K, Othman R. Finite element analysis of adhesively bonded composite joints subjected to impact loadings. Int J Adhes Adhes 2014. http://dx.doi.org/10.1016/j.ijadhadh.2014.07.012.

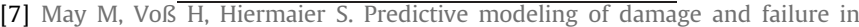
adhesively bonded metallic joints using cohesive interface elements. Int J Adhes Adhes 2014;49:7-17.

[8] Pang SS, Yang C, Zhao Y. Impact response of single lap composite joints. Comput Eng 1995;5:1011-27.

[9] Sato C. Dynamic stress responses at the edges of adhesive layers in lap strap joints of half-infinite length subjected to impact loads. Int J Adhes Adhes 2009;29:670-7.

[10] Da Silva L, Das Neves P, Adams R, Spelt J. Analytical models of adhesively bonded joints - part I: literature survey. Int J Adhes Adhes 2009;29:319-30.

[11] Volkersen O. Die Nietkraftverteilung in zugbeanspruchten Nietverbindungen mit konstanten Laschenquerschnitten. Luftfahrtforschung 1938;15:41-7.

[12] De Bruyne NA. The strength of glued joints. Aircr Eng 1944;16:115-8.

[13] Goland M, Reissner E. The stresses in cemented joints. J Appl Mech 1944;66A:17-27.

[14] Oplinger DW. Effects of adherend deflections in single-lap joints. Int J Solids Struct $1944 ; 31: 2565-87$.
[15] Hart-Smith LJ. Adhesive-bonded single lap joints. 1973 (NASA-CR 112236).

[16] Tsai MY, Oplinger DW, Morton J. Improved theoretical solutions for adhesive lap joints. Int J Solids Struct 1998;35:1163-85.

[17] Vaziri A, Hamidzadeh HR, Nayeb-Hashemi H. Dynamic response of adhesively bonded single-lap joints with a void subjected to harmonic peeling loads. J Multibody Dyn 2001;215:199 (06).

[18] Vaziri A, Nayeb-Hashemi H, Hamidzadeh HR. Experimental and analytical investigation of the dynamic response of a lap joint subjected to harmonic peeling loads. J Vib Acoust 2004;126:84-91.

[19] Vaziri A, Nayeb-Hahsemi H. Dynamic response of the tubular joint with an annular void subjected to a harmonic torsional loading. J Multibody Dyn 2002;216:367-70.

[20] Vaziri A, Nayeb-Hashemi H. Dynamic response of tubular joints with an annular void subjected to a harmonic axial load. Int J Adhes Adhes 2002;22:367-73.

[21] Challita G, Othman R. Analytical model of the double-lap bonded joints response to harmonic loads. Eur J Mech A/Solids 2012:34:149-58.

[22] APC2 PEEK thermoplastic polymer, technical data sheet, Cytec Engineering Materials, http://www.cytec.com/sites/default/files/datasheets/APC-2 PEEK_031912-01.pdf. 